\title{
Détermination de l'épaisseur des glaciers de Sarenne (Alpes) et de Chacaltaya (Bolivie) par prospection radar au sol à $50 \mathrm{MHz}$
}

\author{
Determination of the Sarenne and Chacaltaya glaciers thickness \\ using ground penetrating radar at $50 \mathrm{MHz}$
}

\author{
par Marc Descloitres ${ }^{1}$, Roger Guérin ${ }^{2}$, Edson Ramirez ${ }^{3}$, Robert Gallaire ${ }^{1}$, Pierre Ribstein ${ }^{1}$, François Valla ${ }^{4}$ \\ 1 IRD (Institut de Recherche pour le Développement, ex-ORSTOM), Programme NGT \\ 2 Université Pierre et Marie Curie (Paris 6), UMR 7619 Sisyphe \\ 3 IHH (Instituto de Hidráulica e Hidrología), UMSA, La Paz, Bolivie \\ ${ }^{4}$ CEMAGREF - Etna, Grenoble.
}

Geophysical survey have been conducted on the temperate glaciers of Sarenne (Alps) and Chacaltaya (Bolivia) using Ground Penetrating Radar (GPR) at medium frequency range $(50 \mathrm{MHz})$, in order to evaluate the ice thickness. The results clearly show the reflection at the top of the bedrock at depths over 40 metres for Sarenne. For Chacaltaya, the ice thickness reaches 16 metres only. However, if intermediate reflectors are present, they can attenuate or even overshadow the deep reflection on the bedrock. Those reflectors are attributed to some blocks of rock, crevasses or liquid water. Except for these restrictions, those surveys confirm the interest of GPR at $50 \mathrm{MHz}$ to detect quickly and accurately the bedrock topography for this type of temperate glacier when the thickness remains low.

\section{INTRODUCTION}

La détermination de l'épaisseur des glaciers est importante pour le glaciologue, en particulier lorsqu'il s'agit de connaître les volumes de glace mis en jeu dans les bilans de masse et pour le positionnement des carottages profonds. Certaines méthodes géophysiques permettent cette détermination. Les plus utilisées sont la gravimétrie, la sismique réfraction, la prospection électrique et le radar géologique. Cette dernière méthode recueille les faveurs de la plupart des prospecteurs car elle est relativement facile à mettre en œuvre et fournit des résultats rapidement exploitables. La glace est en effet un milieu où les ondes électromagnétiques radar $(1 \mathrm{MHz}-1 \mathrm{GHz})$ se propagent aisément. La fréquence de prospection est un paramètre essentiel : la plupart des prospections privilégient une grande profondeur d'investigation en utilisant des radars basse fréquence $(1-10 \mathrm{MHz})$ et particulièrement lorsque les glaciers sont tempérés, car les ondes basse fréquence pénètrent plus profondément et sont moins sensibles à la présence d'eau liquide. L'objet des prospections présentées ici dans le cadre du programme "Neige et Glaciers Tropicaux" (NGT) de l'IRD est de montrer que l'utilisation de moyennes fréquences $(50 \mathrm{MHz}$ dans le cas présent) permet d'obtenir des images radar plus fines dans certaines conditions. Elles apportent, depuis la surface, une information non seulement sur l'épaisseur du glacier lorsque celui-ci reste peu épais $(<50 \mathrm{~m})$, mais aussi sur la présence de crevasses de blocs de roche ou eau liquide en profondeur au sein du glacier, ce qui peut aussi masquer l'information sur la profondeur.

Les résultats présentés dans cette étude concernent des prospections entreprises sur les glaciers de Sarenne (Alpes) et de Chacaltaya (Bolivie). Ils mettent en relief les avantages et les limites de l'utilisation du radar de moyenne fréquence pour ces glaciers tempérés.

\section{II — QUELQUES ÉLÉMENTS SUR LA MÉTHODE DU RADAR GÉOLOGIQUE}

\subsection{Généralités}

La méthode géophysique de prospection radar a plusieurs appellations : radar géologique, géoradar, Ground Penetrating Radar (GPR), radar-sol, ou encore " echo-sounding ".

Les équipements commercialisés aujourd'hui sont des radars de type impulsionnel, pour lesquels l'impulsion d'énergie initiale est transmise à l'antenne qui émet selon 
une fréquence centrale donnée, qui dépend de sa géométrie. D'autres types de radar sont actuellement à l'étude, comme les radars à synthèse de fréquence, générant de multiples fréquences en même temps.

Pour les systèmes impulsionnels qui nous concernent ici, les antennes sont considérées comme des dipôles électriques. Ces antennes génèrent à l'émission de l'impulsion un champ électromagnétique d'amplitude variable en fonction du temps et de la distance parcourue. Les équations de Maxwell permettent de décrire le comportement du champ électromagnétique $E$ dans un milieu homogène et isotrope. La loi de propagation selon l'axe vertical des profondeurs $Z$ est :

\section{$\partial^{2} E / \partial Z^{2}-k^{2} E(Z)=0$}

La constante de propagation $k$ est fonction des caractéristiques du milieu dans lequel l'onde est émise. L'expression de $k$ est :

$$
k^{2}=-\varepsilon \mu \omega^{2}+i \sigma \mu \omega
$$

où : $\quad \omega=2 \pi f$, avec $f$, fréquence de l'onde en $\mathrm{Hz}$, $\mu$, perméabilité magnétique. Pour un milieu non magnétique, $\mu=\mu_{0}$, perméabilité magnétique du vide $\left(\mu_{0}=4 \pi 10^{-7} \mathrm{H} / \mathrm{m}\right)$,

$\sigma=$ conductivité électrique du milieu, en $\mathrm{S} / \mathrm{m}$,

$\varepsilon=$ permittivité diélectrique du milieu. D'une manière générale, on a :

$\varepsilon=\varepsilon_{0} \varepsilon_{\mathrm{r}}$ où $\varepsilon_{0}$ est la permittivité dans le vide $\left(\varepsilon_{0}=8,85.10^{-12} \mathrm{~F} / \mathrm{m}\right)$ et $\varepsilon_{\mathrm{r}}$ est la permittivité diélectrique relative du milieu, appelée aussi constante diélectrique $\left(1 \leq \varepsilon_{\mathrm{r}} \leq 80\right)$.

A haute fréquence, comme c'est le cas des ondes radar, le terme $-\varepsilon \mu \omega^{2}$ de la constante de propagation devient prépondérant, et c'est par conséquent la permittivité diélectrique $\varepsilon$ qui gouverne principalement la propagation des ondes dans le sol.

Le tableau I recense les valeurs des paramètres physiques de différents milieux.

La glace est un milieu favorable à la propagation des

\begin{tabular}{|l|c|c|c|c|}
\hline \multicolumn{1}{|c|}{ Milieux } & $\varepsilon_{\mathrm{r}}$ & $\sigma\left(\mathrm{mS} \cdot \mathrm{m}^{-1}\right)$ & $v\left(\mathrm{~m}^{\left.-\mathrm{ns}^{-1}\right)}\right.$ & $\alpha\left(\mathrm{dB} \cdot \mathrm{m}^{-1}\right)$ \\
\hline Air & 1 & 0 & 0,30 & 0 \\
\hline Glace & $\mathbf{3 - 4}$ & $\mathbf{0 , 0 1}$ & $\mathbf{0 , 1 6}$ & $\mathbf{0 , 0 1}$ \\
\hline Eau distillée & 80 & 0,01 & 0,033 & 0,002 \\
\hline Eau douce & 80 & 0,5 & 0,033 & 0,1 \\
\hline Eau salée & 80 & 3000 & 0,01 & 1000 \\
\hline Sable sec & $3-5$ & 0,01 & 0,15 & 0,01 \\
\hline Sable humide & $20-30$ & $0,01-1$ & 0,06 & $0,03-0,3$ \\
\hline Calcaire & $4-8$ & $0,5-2$ & 0,12 & $0,4-1$ \\
\hline Marnes & $5-15$ & $1-100$ & 0,09 & $1-100$ \\
\hline Argiles & $5-40$ & $2-1000$ & 0,06 & $1-300$ \\
\hline Granite & $4-6$ & $0,01-1$ & 0,13 & $0,01-1$ \\
\hline Sel (halite) & $5-6$ & $0,01-1$ & 0,13 & $0,01-1$ \\
\hline
\end{tabular}

Tableau I. Principaux paramètres pour le radar géologique (d'après [3]). ( $v$ et $\alpha$ sont respectivement la vitesse de propagation des ondes radar, et le coefficient d'atténuation). ondes radar grâce à sa faible permittivité diélectrique relative, sa faible conductivité et par le fait que l'atténuation y reste faible.

Le lecteur pourra se reporter aux publications de Ulriksen [1], Tillard [2], Annan [3] ou Reynolds [4] pour une description exhaustive des principes fondamentaux qui régissent la propagation des ondes radar et la méthode de prospection proprement dite.

\section{- 2.2 Equipement et mise en œuvre}

Le radar utilisé dans cette étude est un Pulse Ekko 100 du constructeur Sensors and Software. Il est constitué d'une unité centrale d'acquisition reliée à un ordinateur portable. L'unité centrale est reliée aux antennes d'émission et de réception par des câbles en fibre optique garantissant une transmission des données sans atténuation notable. L'ensemble pèse moins de $20 \mathrm{~kg}$.

La mise en œuvre est illustrée sur la figure 1. L'ensemble est manipulé par 3 personnes en terrain difficile, mais peut être monté sur un traîneau et tiré par un seul opérateur en terrain dégagé. Pour notre étude, nous rappelons que la fréquence est de $50 \mathrm{MHz}$. Une impulsion est générée tous les $0,5 \mathrm{~m}$, et un profil de $500 \mathrm{~m}$ est effectué en $2 \mathrm{~h}$ environ. Pour chaque émission, l'onde se propage en profondeur jusqu'à rencontrer une interface réfléchissante (bedrock, hétérogénéités dans la glace, rochers, crevasses ou eau liquide par exemple), puis est renvoyée vers le récepteur où elle est enregistrée.

\subsection{Représentation des résultats}

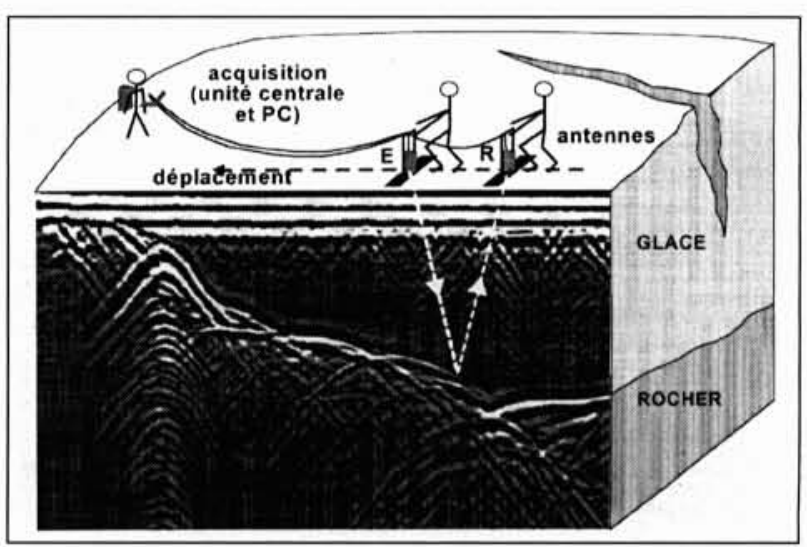

1. Mise en æuvre du radar sur un glacier.

Les résultats sont représentés sur un radargramme, graphique sur lequel chaque trace acquise est juxtaposée avec la précédente sur l'axe des abscisses selon le temps en ordonnée. On peut convertir le temps en profondeur connaissant la vitesse de propagation des ondes dans la glace. Cette vitesse est en général proche de $0,16 \mathrm{~m} / \mathrm{ns}$ (soit un peu plus que la moitié de la vitesse de la lumière dans le vide qui est de $0,30 \mathrm{~m} / \mathrm{ns}$ ).

Plusieurs procédés graphiques permettent d'accentuer les arrivées d'énergie correspondant aux différentes réflexions qui se produisent dans le sous-sol. Dans le cas d'un glacier, le réflecteur principal est constitué par la roche sous-jacente. Cependant, si des couches ou des objets réflecteurs se trouvent entre la surface et la roche, les réflexions correspondantes apparaissent moins profondément sur le radargramme et masquent parfois totalement la réflexion sur la roche. 


\section{III — RÉSULTATS}

\section{- 3.1 Glacier de Sarenne}

Le glacier de Sarenne, situé dans le massif des Grandes Rousses (fig. 2), a été prospecté par radar basse fréquence $(1,25$ à $10 \mathrm{MHz})$ par Funk et al. [5]. Cette prospection a permis d'estimer son épaisseur. Ce glacier constitue un site test pour notre équipement.

Le radargramme du profil 2 (fig. 2) est présenté sur la figure 3. Dans la partie amont du profil, la roche est clairement repérée à moins de $30 \mathrm{~m}$ de profondeur. A partir de l'abscisse $300 \mathrm{~m}$, le substratum s'approfondit brusquement. L'image radar est ensuite brouillée par de multiples réflexions intervenant avant la roche et qui finissent par la masquer totalement.

Ces réflexions multiples ont probablement pour origine des crevasses invisibles en surface, ou des hétérogénéités (rochers, couches de glace " sale", eau liquide en quantité importante). Différents traitements ont été menés de manière à faire ressortir le bedrock au-delà de $40 \mathrm{~m}$ de profondeur. Par exemple, un filtrage spatial basse fréquence (fig. 3) fait apparaître une continuité latérale de plus forte amplitude pouvant être suivie au centre du profil vers $45 \mathrm{~m}$ de profondeur. Il pourrait s'agir du réflecteur correspondant au substratum mais la prospection de Funk et al. [5] le situe vers $60 \mathrm{~m}$ dans cette zone. Les basses fréquences sont en effet moins influencées par les petits objets. Dans notre cas des moyennes fréquences, les ondes sont plus souvent arrêtées par les hétérogénéités. Il est possible que le filtrage ait fait apparaître une continuité factice entre plusieurs réflecteurs discontinus. Quoiqu'il en soit, ce résultat met en évidence une des limites de l'emploi des moyennes fréquences lorsque le glacier comporte beaucoup de réflecteurs interposés

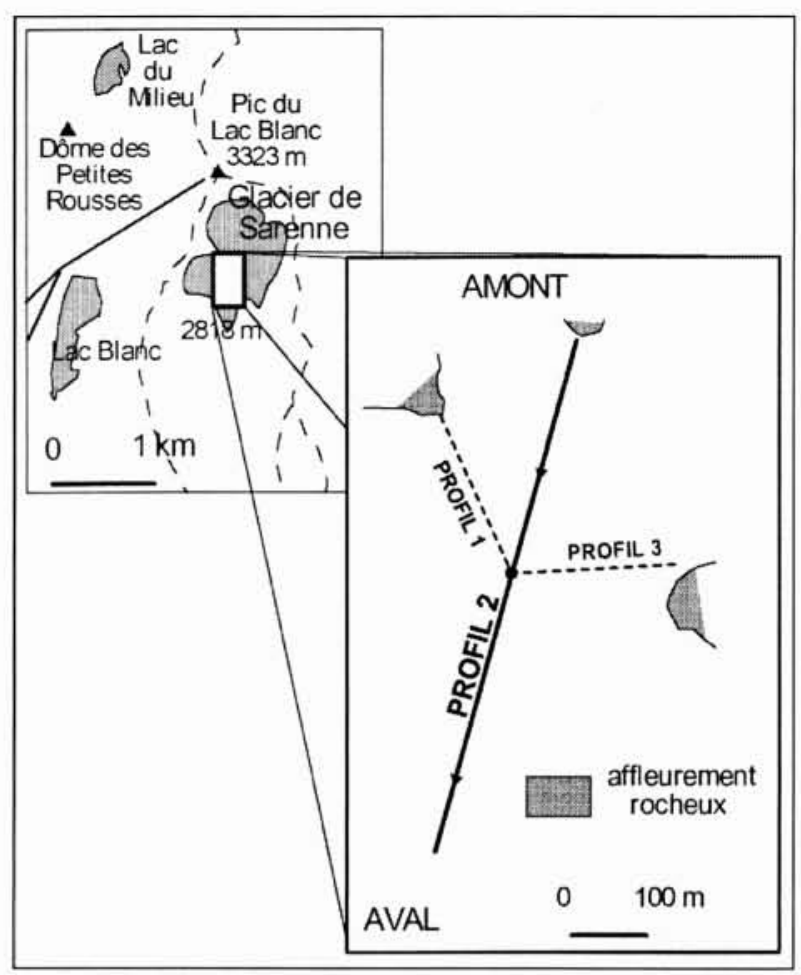

2. Localisation du glacier de Sarenne et positions des profils radar.

entre la surface et la roche et particulièrement, la présence d'eau liquide pourrait être le facteur gênant le plus important pour la propagation. Dans le cas présent, la roche n'est clairement repérée que jusqu'à $40 \mathrm{~m}$.

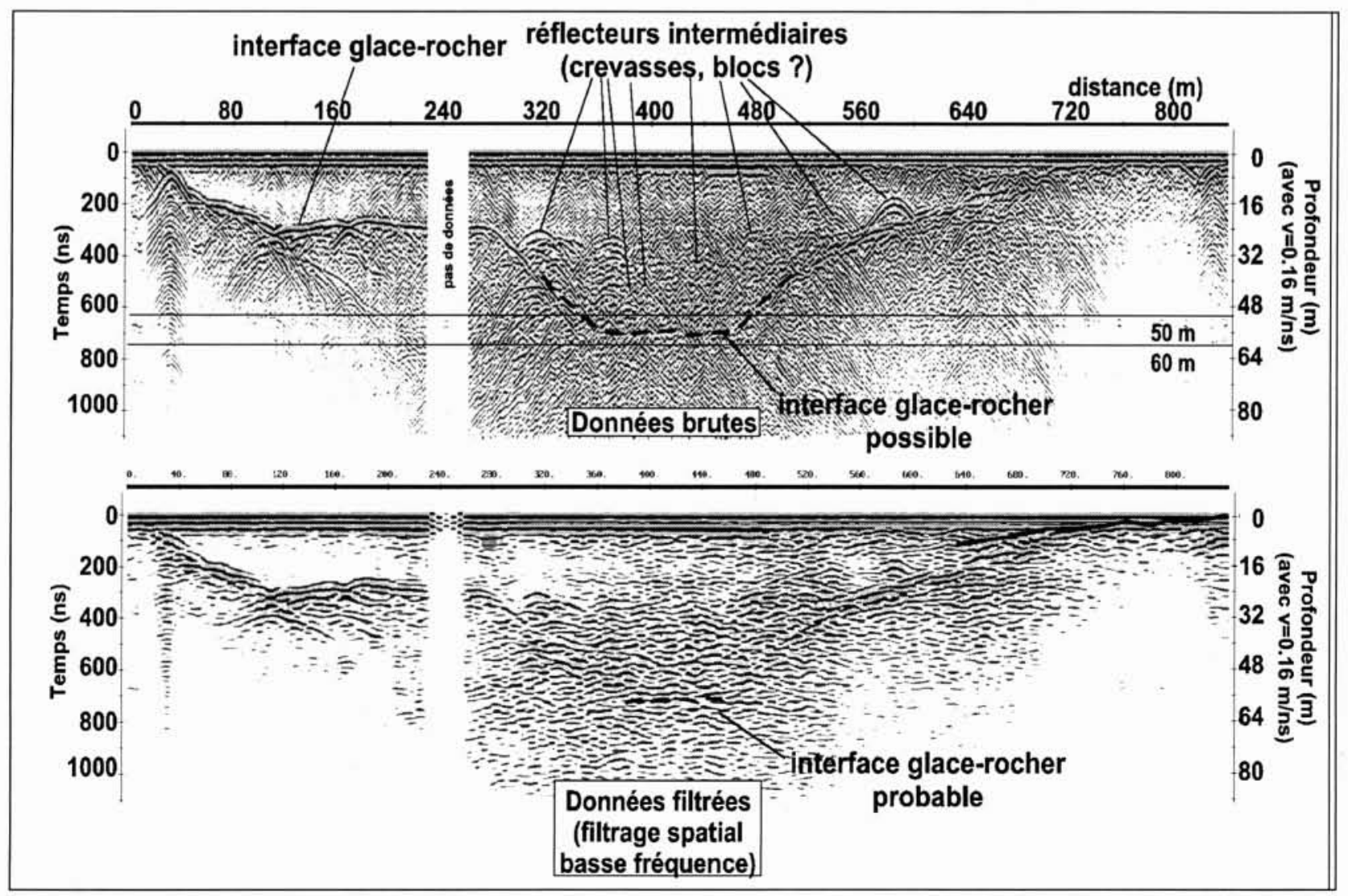

3. Glacier de Sarenne. Radargramme à $50 \mathrm{MHz}$ du profil 2, données brutes et filtrées. 


\subsection{Glacier de Chacaltaya}

Situé dans la Cordillère Royale en Bolivie, le glacier de Chacaltaya domine la ville de $\mathrm{La}$ Paz et constitue, par sa petite taille, un témoin idéal pour évaluer l'influence des variations climatiques sur les bilans de masse de tous les glaciers environnant cette capitale. Ces glaciers représentent en effet environ $70 \%$ de l'alimentation en eau potable de la ville. $\mathrm{La}$ figure 4 présente l'évolution de la surface du glacier de Chacaltaya depuis 1850. C'est un glacier de cirque, entre 5123 et 5375 mètres d'altitude. Sa longueur est de 584 mètres, et sa superficie de $0,082 \mathrm{~km}^{2}$. Dans le cadre du programme NGT, le bilan de masse est suivi depuis 1991 grâce à des mesures mensuelles. Ces mesures font apparaître un bilan plutôt négatif, tandis que le glacier a fortement reculé [6].

La détermination précise de son épaisseur prend dans ce contexte une signification particulière : " jusqu'à quand pourra-t-on compter sur ce glacier, et ses voisins, pour alimenter en eau la ville et les retenues des centrales hydroélectriques ? " est une des questions à laquelle tentent de répondre les glaciologues. La prospection radar entreprise en 3 jours comporte 10 profils ( 2 longitudinaux et 8 transversaux) situés sur la figure 4 . Les radargrammes correspondants sont présentés sur la figure 5 en perspective, sans que la topographie de la surface du glacier, très bombée, soit prise en compte.

La vitesse dans la glace a été estimée par traitement (migration). Elle est de $0,155 \mathrm{~m} / \mathrm{ns}$, avec une incertitude estimée à

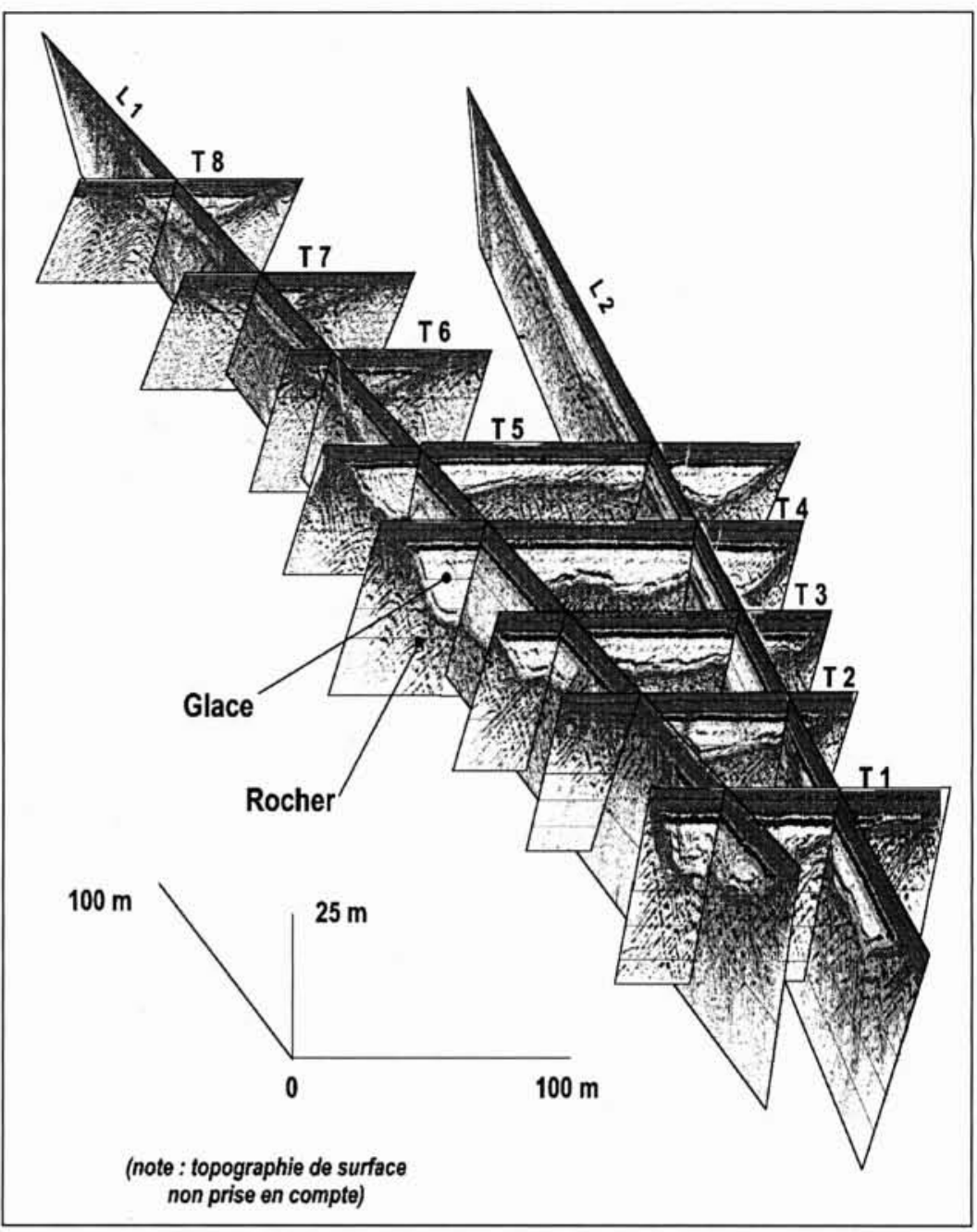

5. Glacier de Chacaltaya. Radargrammes 50 MHz. Représentation en perspective.

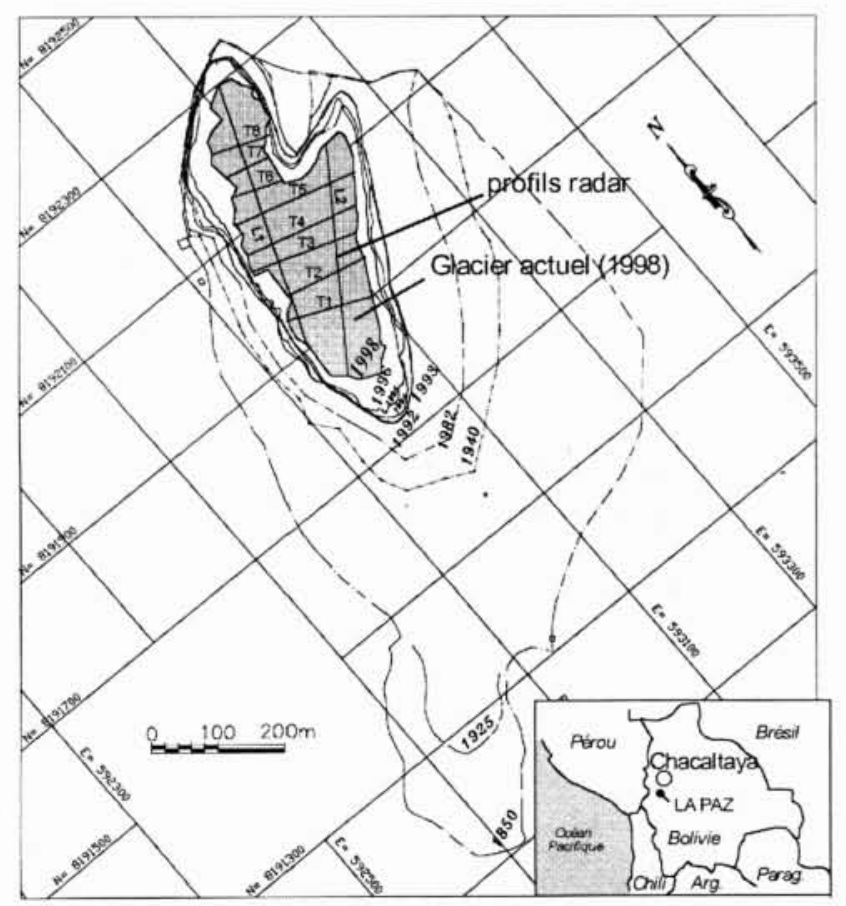

4. Glacier de Chacaltaya. Situation, évolution de la surface depuis 1850 et position des profils radar.
$0,005 \mathrm{~m} / \mathrm{ns}$. Cette incertitude peut conduire à une variation dans la valeur de l'épaisseur calculée : par exemple, pour un temps de trajet de $200 \mathrm{~ns}$, l'épaisseur calculée avec des vitesses de 0,15 et $0,16 \mathrm{~m} / \mathrm{ns}$ est respectivement de 15 et 16 mètres. La profondeur maximum de la roche repérée sur les profils recoupant le centre du glacier est de $16 \mathrm{~m}$. Dans le cas de Chacaltaya, aucune réflexion "parasite" ne vient perturber l'image, bien que certains profils fassent apparaître des crevasses ponctuellement.

Les erreurs sur la détermination de la profondeur peuvent avoir plusieurs origines : géométriques (trajet des ondes déformé), graphiques (mauvaise estimation de l'arrivée d'énergie), intrinsèques à la fréquence utilisée, ou dues à une mauvaise détermination de la vitesse. Pour cette prospection, nous avons estimé l'erreur possible à $\pm 0,5 \mathrm{~m}$.

Le pointé de la réflexion sur les radargrammes permet de construire une carte d'épaisseur du glacier. Connaissant la topographie de surface et l'épaisseur selon un pas régulier en distance le long des profils, il s'agit de transcrire les coordonnées relatives des points de mesure dans le système de coordonnées principales des topographes. La carte d'épaisseur présentée sur la figure 6 est le résultat de ce traitement. Elle fait clairement apparaître un épaississement du glacier dans la partie centrale du profil longitudinal 1 alors que dans la partie centrale du glacier l'épaisseur dépasse rarement 8 mètres. 
Le volume total de glace est estimé à $420000 \mathrm{~m}^{3}$ \pm 25000 . Sachant que le glacier fond de $1 \mathrm{~m}$ d'épaisseur par an, il reste un peu plus d'une dizaine d'années avant la disparition complète du glacier.

Nous mentionnons aussi qu'une prospection similaire a été menée sur le glacier voisin du Zongo, beaucoup plus épais et enchâssé entre des parois instables, ce qui provoque de nombreux éboulements : la prospection à $50 \mathrm{MHz}$ n'a pas révélé le bedrock au-delà de 50 mètres, en raison des innombrables réflexions provoquées par des rochers éboulés, prisonniers de la glace et ce, sur des épaisseurs probablement très importantes. Cela confirme la limite de l'emploi des moyennes fréquences dans ce cas, à l'image de la prospection sur Sarenne.

\section{IV - CONCLUSIONS}

Les prospections radar entreprises sur des glaciers des Alpes et des Andes Boliviennes permettent de confirmer l'intérêt des images radar à $50 \mathrm{MHz}$ pour la détection précise de la roche sous-jacente et des crevasses, rochers ou eau libre au sein de la glace et invisibles depuis la surface. Cependant, lorsqu'il existe de nombreuses hétérogénéités au sein de la glace, cela peut masquer l'information du bedrock. Dans ce cas, des équipements radar basse fréquence (1$10 \mathrm{MHz}$ ) devront être préférés, en particulier en présence de glaciers tempérés très épais. Les images à $50 \mathrm{MHz}$ trouveront aussi leur plein intérêt dans le cas de prospections sur des glaciers froids. La précision des images obtenues avec un pas de mesure très fin autorise aussi des traitements analogues à ceux conduits sur les coupes sismiques pour l'exploration pétrolière. Il est probable que des zones contenant de l'eau liquide, ou d'accumulations de débris ou de moraines, puissent être identifiées par des traitements appro-

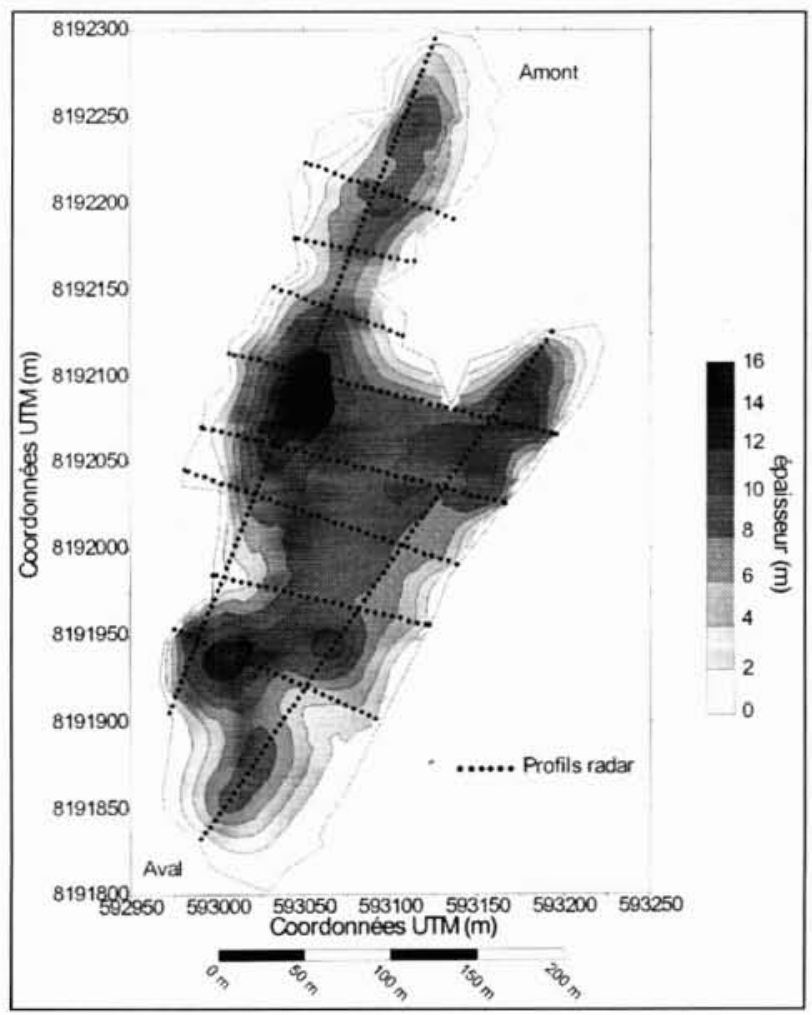

6. Glacier de Chacaltaya. Epaisseur de glace déduite de l'interprétation radar. priés. Ces perspectives, déjà esquissées dans certaines publications, demandent néanmoins à être confirmées sur des glaciers bien connus.

\section{REMERCIEMENTS}

Cette étude a été réalisée dans le cadre du projet Neige et Glaciers Tropicaux de l'IRD (anciennement ORSTOM). Les auteurs remercient vivement toutes les personnes qui ont contribué aux prospections. A Sarenne, toute l'équipe du CEMAGREF - Etna, à Chacaltaya et Zongo, J.P. Chazarin et J.E. Sicart (IRD, programme NGT), J. Mendoza (IHH, La Paz), L. Noriega (SENAMHI, La Paz).

\section{RÉFÉRENCES :}

[1] ULRIKSEN, C.P., 1982. Application of impulse radar to civil engineering. Doctoral thesis of Lund University, Sweden. Thèse rééditée par le constructeur Geophysical Survey Systems Inc., 13 Klein Drive, North Salem, N.A., 03073 USA.

[2] TILLARD, S., 1988. Evaluation de la prospection radar en prospection géophysique. Approche théorique et expérimentale. Mémoire d'ingénieur de l'EOPGS, Strasbourg.

[3] ANNAN, P.A., 1992. Ground penetrating radar. Workshop notes. Publication sur demande auprès de Sensors and Software Inc, 1091 Brevik Place, Mississauga, Ontario, L4W3R7, Canada.

[4] REYNOLDS, J.M., 1997. An introduction to Applied and Environmental Geophysics. John Wiley and Sons Ltd, Chichester, UK. (Csbooks@wiley.co.uk)

[5] FUNK, M., BÖSCH, H. et VALLA, F., 1993. Mesures des épaisseurs de glace par méthode radar au glacier de Sarenne. Rapport interne Ecole Polytechnique Fédérale de Zürich et CEMAGREF Grenoble.

[6] FRANCOU, B., RAMIREZ, E., MENDOZA, J., GUERECA, J. MIRANDA, G. y NORIEGA, L., 1998. El glaciar de Chacaltaya, Investigaciones glaciologicas 1991-1997. Informe 56, Orstom, La Paz.

\section{Bibliographie non exhaustive : pour en savoir plus...}

[7] ARCONE, S.A., 1996. High resolution of glacial ice stratigraphy : a ground penetrating radar study of Pegasus Runway, McMurdo Station. Antartica. Geophysics, 61, 6, 1653-1663.

[8] BJORNSSON, H., GJESSING, Y., HAMRAN, S., HAGEN, J.O., LIES TOL, O., PALSON, F, and ERLINGSSON, B., 1996. The thermal regime of sub-polar glaciers mapped by multi-frequency radio-echo sounding. Journal of Glaciology, 42, 140, 23-32.

[9] CORR, H., MOORE, J.C. and NICHOLLS, K.W., 1993. Radar absorption due to impurities in antartic ice. Geophysical Research Letters, 20. 11, 1071-1074

[10] HAMRAN, S.E., AARHOLT, E., HAGEN, J.O. and MO, P., 1996. Estimation of relative water content in a sub-polar glacier using surfacepenetration radar. Journal of Glaciology, 42, 142, 533-537.

[11] MOORE, J.C. and FUITA, S., 1993. Dielectric properties of ice containing acid and salt impurity at microwave and low frequencies. Journal of Geophysical Research, 98, B6, 9769-9780.

[12] THOMPSON, L.G., 1992. Interpretation of short-pulse radar soundings from low latitude, high altitude glaciers of Peru and China. In Ground penetrating radar, ed. J. PILON ; Geological Survey of Canada, paper 90.4. 213-225. 